\title{
Design and Development of Intelligent Riding Assistant Equipment Based on Attitude Recognition
}

\author{
Jiaxin Tian ${ }^{1, a}$, Yang Du ${ }^{2, b}$, Chenzhi Tang ${ }^{3, c}$ and Fei Guo ${ }^{4, d^{*}}$ \\ Information Engineering Institute, Beijing Institute of Fashion Technology, Beijing, China \\ a617724382@qq.com, b18310236998@163.com, '2542122034@qq.com, dunwen95@163.com
}

Keywords: Arduino; Cycling; Safety; Neural network.

\begin{abstract}
This program mainly use the neurons in the main control board and six-axis acceleration sensor belong to Intel based on Arduino called Curie Nano to collect the gesture data. And after extracted the feature of these data, motion data training must be begun, then to recognize the relevant motion such as left and right, in order to lighten the relevant LED light. For this result, drivers can be warmed to take evasive action to reduce the traffic accident. Through the test controller really wear it and then to test in the relevant area. As the result, the recognition rates of left is $92 \%$, and the right is $96 \%$. So, left and right can be better recognized.
\end{abstract}

\section{Introduction}

With the popularity of shared bicycles, Whether on the university campus, on the way to get off work, or go out on the way to play, the cycling team can be found everywhere. For this economical and convenient, low carbon green travel mode, it is beneficial to people's physical and mental health, but also improve the efficiency of travel[1]. However, because of the number of sharing bicycles and riders increasing, and for the complex city traffic condition, the safety of riders is more and more noticed by society.

Based on the above background, an intelligent assistant equipment for cycling has been designed in this program. LED light is added on the comfortable breathable fabric for riding suits, and they are controlled through the six axis attitude sensor and gyroscope set on the spinal position of the cycling personnel. When turning right or left, the LED light which is in relevant sleeve would be lighten. Through above measures, the riding intention of rider would be delivered to other motor vehicles and pedestrian, in order to remind them to take relevant steps to avoid that, so it can promote the safety factor especially in the night.

\section{Design Plan}

\section{A. Functions of Designing}

This program is based on open source electronic prototyping platform and compatible hardware modules for Arduino. Using the six-axis acceleration sensor and gyroscope to collect data and pretreatment, features of these data would be extracted[2]. After that, convergence of data must be judged through using MATLAB. Finally, these data after processing would be delivered to the main control board. Inside this board, there are 128 neurons to study and training. Bluetooth serial port can keep track of delivery condition for real time data[7].

The program of real operation is extremely easy. Before the rider turn, he should move his shoulder a little to the relevant direction. Then the sign light settled on arms of rider would be twinkled to remind drivers and pedestrian to watch out. Just needing some small amplitude, this program can reduce the dangers of riding. 


\section{B. Appearance Design}
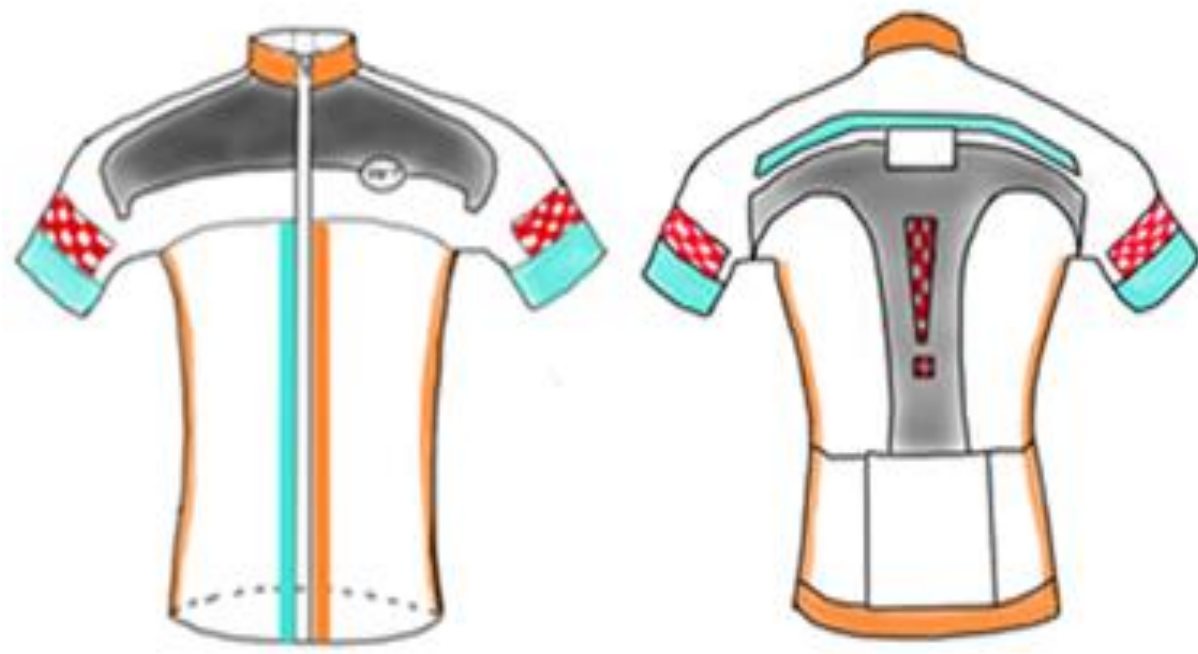

Figure 1. Indicator Lamp Appearance.

Reseau in arms is the position of sign light. Square frame in back is the position of controller.

The sign light LED in arms are around of whole upper-arm circumference, and different drivers and pedestrians all can watch this sign light.

\section{BP Neural Network}

\section{A. Artificial neuron}

Figure 2 is the theory of artificial neuron.

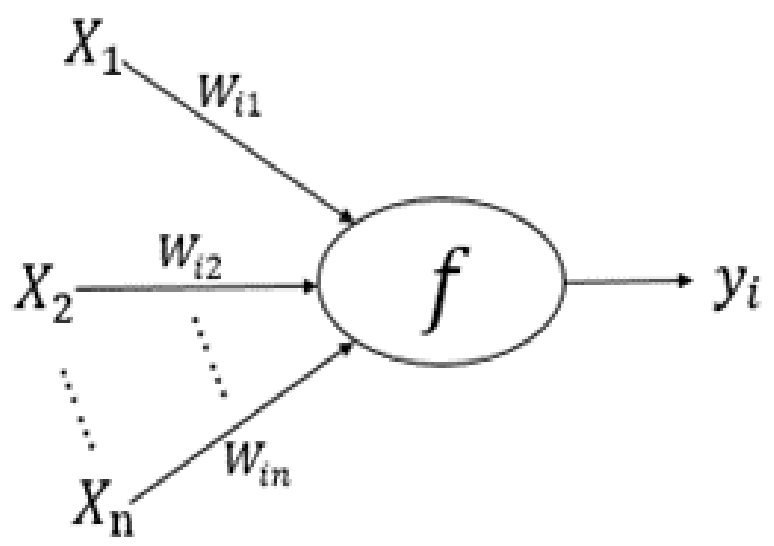

Figure 2. Theory of Artificial Neuron.

In the figure above, $\mathrm{X} 1, \mathrm{X} 2, \ldots \ldots, \mathrm{Xn}$ called input sign are sent by other neurons. And $\mathrm{Wi1}, \mathrm{Wi} 2, \ldots \ldots$. Win called weights is delivered between input layer and hidden layer.

The input or output relation of Neural numbered $i$ is as follows:

$$
\text { net }_{i}=\sum_{j=1}^{n} w_{i j} x_{j}-\theta
$$

$$
y_{i}=f\left(\text { net }_{i}\right)
$$

In formula above, net is called the net activation, $\theta$ is represented for threshold, $\mathrm{f}$ is represented for active function, and yi is called for neuron number $\mathrm{i}$. If offset value is used as one of the weights for neuron numbered $i$, the function above could be written as follows: 


$$
\begin{aligned}
& \text { net }_{i}=\sum_{j=0}^{n} w_{i j} x_{j} \\
& y_{i}=f\left(\text { net }_{i}\right)
\end{aligned}
$$

If using vector to represent relation above, the formula is as follow:

$X=\left[x_{0}, x_{1}, x_{2}, \ldots \ldots, x_{n}\right]$

$$
W=\left[\begin{array}{c}
w_{i 0} \\
w_{i 1} \\
w_{i 2} \\
\cdot \\
\cdot \\
\cdot \\
w_{i n}
\end{array}\right]
$$

Using vector multiplication to represent output of neuron, as follows:

$$
\text { net }_{i}=X W
$$

$$
y_{i}=f\left(\text { net }_{i}\right)=f(X W)
$$

As net activation calculated of neuron is positive, it represents neurons in the active state. When net activation calculated of neuron is negative, relative neuron is controlled[3].

\section{B. BP Neuron Network algorithm}

BP algorithm has super nonlinear mapping ability. In the three layers BP network, It can realize the high order approximation of all nonlinear functions. For this algorithm, a group of motion data needed training is delivered to this network, and compared real output value with expected output value. Through previously compared, there are some D-value, then reducing the D-value gradually. The detail steps are as follow:

a. Taking a sample value in the sample set;

b. Calculating the output value of BP neural network in the actual situation $\mathrm{O}$;

c. Find the difference between $\Delta=\mathrm{Bi}-\mathrm{O}$;

d. According to the weight of delta adjustment matrix W;

e. For each above steps, repeated execution, the error control is within a smaller range relative to the large sample set until the computed results are compared with the large sample set.

\section{BP Neural Network Data Preprocessing}

Data preprocessed can be sent to the network to study and train. In general, data collected would be mapped to a small side range. Because the input data's range might be relatively large even different unit, these data would make some error and cause that the convergence rate of the neural network is reduced. So, normalization algorithm can be achieved by using a brief and rapid linear transformation method.

As the range of active function is section from 0 to $1, y=(x-\min ) /(\max -\min )$ this formula can be used.

As the range of active function is section from -1 to $1, y=2 * \frac{x-\min }{\max -\min }-1$ this formula could be used.

\section{BP Neural Network's Realization by MATLAB}

Neural network is just a model, actually, for its application, tis realization should be complicated through using some measure, such as the built network structure model of MATLAB. Input signal is used as the data for inputting to MATLAB and inputted into the neural network, these data would be calculated through relevant algorithm to handle. Finally, it can achieve the nonlinear processing. And then, using the handled data by MATLAB is used as input quantity again to other neuron and so on, the neural network is formed[4]. 
Using the MATLAB to simulate BP algorithm for acceleration data, in here, only showing a part of data just as follow:

Table 1. Three axis acceleration value of left and right steering

\begin{tabular}{|c|c|c|}
\hline X Axis & Y Axis & Z Axis \\
\hline 0.07 & -0.03 & 1.08 \\
\hline 0.07 & -0.01 & 1.08 \\
\hline 0.07 & 0 & 1.06 \\
\hline 0.07 & 0 & 1.07 \\
\hline 0.06 & -0.01 & 1.11 \\
\hline 0.06 & -0.01 & 1.13 \\
\hline
\end{tabular}

In this table's three column data, they represent the acceleration values of the left and right steering of the $\mathrm{X}$ axis, the $\mathrm{Y}$ axis and the $\mathrm{Z}$ axis respectively.

The collected data is simulated by MATLAB, and the process is as follows:

Read training data $\rightarrow$ eigenvalue normalization $\rightarrow$ construct output matrix $\rightarrow$ create neural network $\rightarrow$ set training parameters $\rightarrow$ start training[5]

The learning interface of the neural network is shown in the diagram:

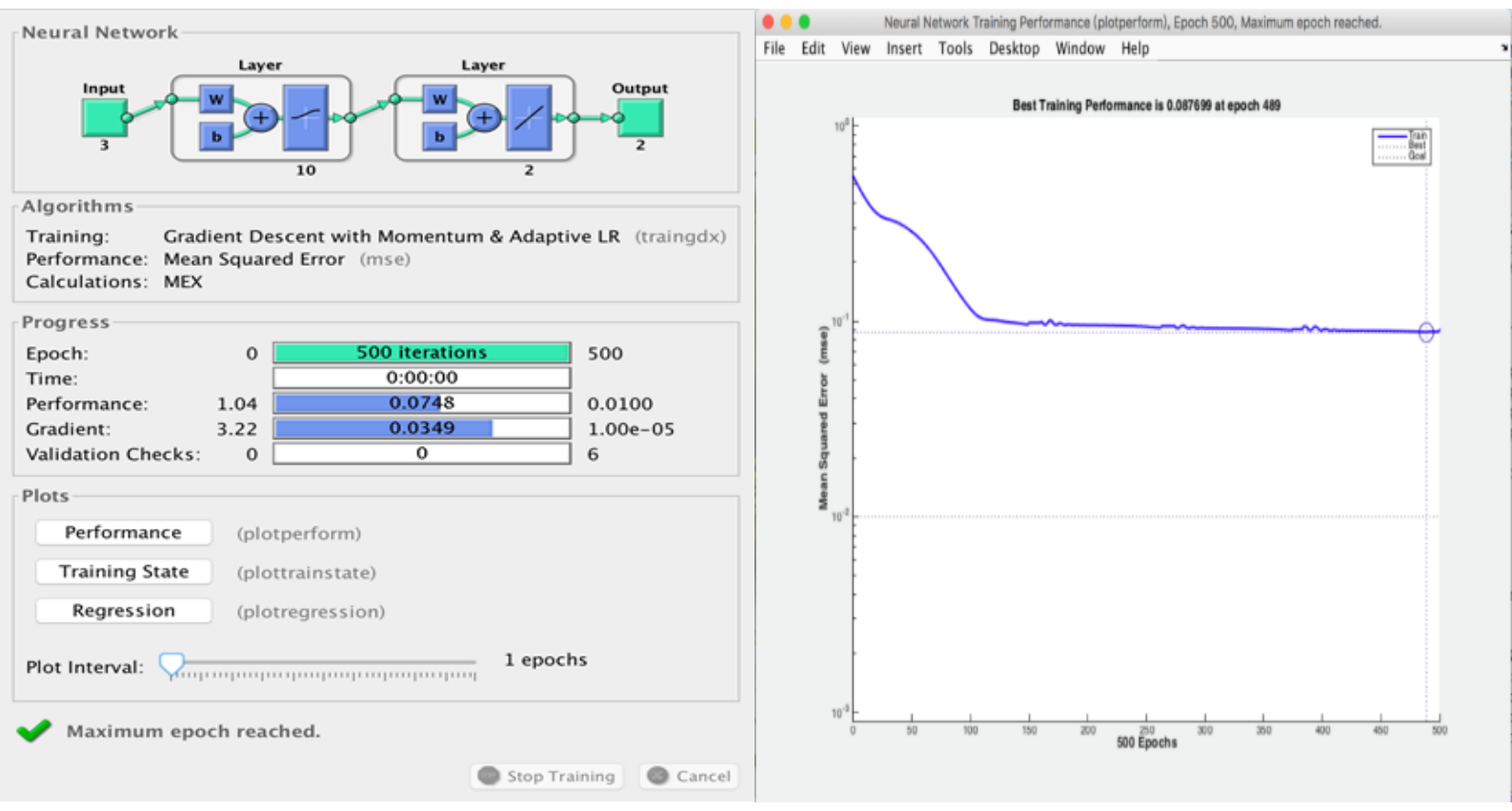

Figure 3. Interface of neural network learning and training by MATLAB.

The figure above shows that the data is convergent, and can be used in BP neural network for data recognition and learning. The acceleration sensor data obtained is disordered in real time, but also has the convergence, so BP neural network algorithm programming this project the main control board is effective on acceleration sensor data acquisition.

\section{Hardware Module and Whole Connection}

\section{A. Introduction of Main Control Panel}

This project with the main control board for the Curie Nano control panel is mini development tool based on Intel Curie module, perfectly compatible with all the performance of Arduino/Genuino 101, 
enhanced the power part and sensor design, the use of relatively simple. It can be perfectly applied in area of wearable because of its highly compositive size. Its appearance is as follow:

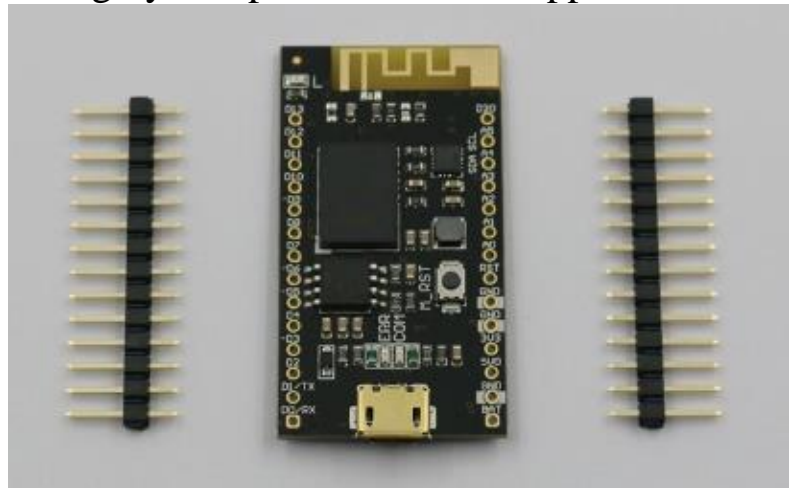

Figure 4. The Main Controlled Board of Curie Nano.

The main controlled chip of Curie Nano is Intel Curie. Inside the module, $34 \mathrm{MHz}$ bluetooth module and six-axis accelerate are settled for clock frequence. It includes 15 digital I/O ports and I/O input port. The module contains 128 hardware neurons, which can be learned by examples without offline programming, and can be maintained only with a small amount of power.

\section{B. Other Hardware Module}

\section{Read write memory module of Arduino TF card}

TF Card is called TransFlash. With TF card initialization and read-write function examples in Arduino IDE built-in SD card program library. TF card between the function block and the core function module of the communication form is done using the SPI bus.

\section{Battery Boost Module}

The AA battery booster module used in this project can boost the voltage of $3 \mathrm{~V}$ connected by 2 dry batteries in series to $5 \mathrm{~V}$, and supply power to the sensor and the experimental circuit. The size is $62 * 33 \mathrm{~mm}$, which effectively reduces the volume of the power supply.

\section{LED 5V Lamp Belt}

The starting voltage of the flexible LED lamp belt is $5 \mathrm{~V}$, which can be lit by the ordinary 5 battery or mobile power supply, and the safety performance is good. The lamp can be made into any shape, and the brightness is high, the use of technology to prevent dead, one does not light does not affect other lamps, can be flexibility in the use of wearable clothing.

The hardware diagram is as follows

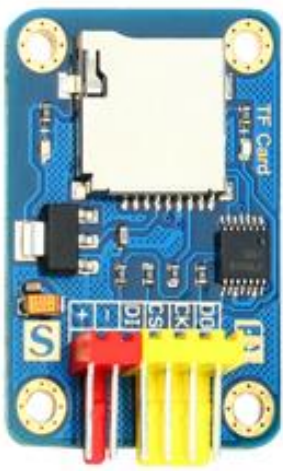

TF module appearance drawing

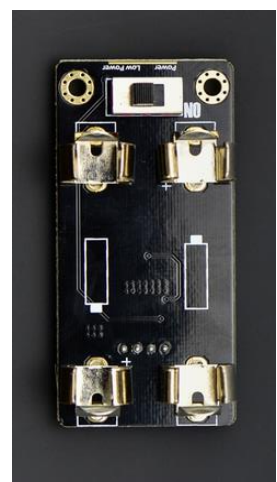

Real diagram of AA battery boost module

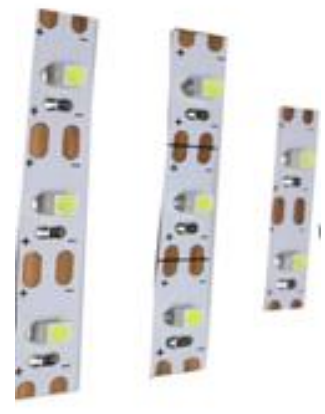

Flexible LED lamp with physical map

Figure 5. Other Hardware Module Appearance Drawing.

\section{Hardware Overall Design}

With the boost module to the main control board and the lamp with power supply, will collect and handle the data values stored in the SD card, with the main control board SD card read information, 
when monitoring the riding personnel to make the set operation, the neural network recognition function, the corresponding position of the cue light flashing[6].

\section{Experimental Results and Summary}

In a complete hardware system, the initial position of the USB interface board Curie down, when a Nano angle offset to the left and left in place, to remind the lights flashing, an angle Nano and offset to the right place, right turn lights can be lit to remind. The actual wear test was performed by the cycling personnel, and the results were shown in table below:

Table 2. Actual Test Results

\begin{tabular}{|c|c|c|c|c|}
\hline Action & Test times & Exact times & Errors' time & Recognition rate \\
\hline Turning Left & 25 & 23 & 2 & $92 \%$ \\
\hline Turning right & 25 & 24 & 2 & $96 \%$ \\
\hline
\end{tabular}

The actual test shows that the right and left steering can be identified more accurately, the recognition rate is higher than $90 \%$, but the identifiable action is larger than the preset range of action, still needing to be improved.

\section{Acknowledgement}

In this paper, the research was sponsored by 2013 National Science and Technology Support Program funded projects (Project No. 2013BAH41F02) and 2017 Beijing Municipal Education Commission Science and Technology Program on the surface of the project (Project No. SQKM201710012006) and Research on the construction of the university library resources recommending platform based on user-driven (Project No. KYJH02160201/002/022) and Innovative design of garment ergonomics and apparel functions Beijing key Laboratory (Project No. KYTG02170202) and Research on the application of mobile learning to the improvement of college students ' creativity and practical ability the key project of education and teaching reform in Beijing Garment College (Project No. ZDJG-1505) and Beijing Key Laboratory of digital interaction and media.

\section{References}

[1] Li Jing bicycle: intelligent riding has been on the road, [N]. consumer daily.2014-11-25 (A02)

[2] Zhao Yingjie. Perfect illustration. Introduction to Arduino interactive design. Beijing: Science Press, 2014.7.

[3] Chen Wenbai. Principle and practice of artificial neural network. Xi'an: Xi'an Electronic and Science University press, 2016.01.

[4] Freescale technology product development center, MATLAB6.5 aided image processing, publishing house of electronics industry, 2003.1

[5] Chen Ming, the principle of neural network and example, Tsinghua University press, 2013.3.

[6] Lu Caiwu, Tang Xiaoling, Zhang Zhixia, Gu Qinghua. Computational intelligence. Xi'an: Shaanxi science and Technology Press, 2008.10.

[7] Sun Dejun, editor. Easy to learn, fun Arduino.: Chemical Industry Press, 2016.10. 